Pacific Journal of Mathematics

REPRODUCING KERNELS AND OPERATORS WITH A 


\title{
REPRODUCING KERNELS AND OPERATORS WITH A CYCLIC VECTOR I
}

\author{
VASHIShTHA N. SINGH
}

In this paper a study is begun of the complete unitary invariant $\left((1-w T)^{-1} e,(1-z T)^{-1} e\right)$, first considered by Livsic in his paper 'On Spectral Resolution of Linear Nonself Adjoint Operators' Mat. Sb., 34 (76), 1954, 145-199, of a triple $(T, H, e)$ where $T$ is a bounded linear operator on a Hilbert space $H$ and $e$ is a cyclic vector for $T$ in $H$, as a reproducing kernel. One of the important points is the construction of a subset of the group algebra of the torus closed under pointwise addition and convolution. This obviously will generate a ring called the $K$-ring. A study of this ring will be done later.

Several other theorems and constructions are also given.

Introduction. Let $T$ be a bounded linear operator on a Hilbert space $H$ with a topologically cyclic vector $e$ in $H$. In this paper we wish to study certain analytic functions associated with the triple $(T, H, e)$ for the sake of the problem of invariant subspaces of $T$ in $H$. (See also [8] and [15].)

The paper is divided into six sections. In $\S 1$ we present some facts about reproducing kernels with analyticity properties. In $\S 2$ we consider a triple $(T, H, e)$ of the above type. $H$ can then be represented as a Hilbert space of conjugate analytic functions $\alpha_{e}[H]$ with a reproducing Kernel $K . T^{*}$ on $H$ assumes the form of reverse shift on the Taylor coefficients of functions in $\alpha_{e}[H]$. (See also [11] or [19].) In $\S 3$ we recover $(T, H, e)$ from the reproducing kernel of $\alpha_{e}[H]$ in two ways. The notion of an analytic function of positive definite type is introduced and it is shown that only these can arise as reproducing kernels of $\alpha_{e}[H]$. These functions are also related to invariant subspaces $(\S 4)$. In $\S 5$ a category of triples is constructed and it is connected to the harmonic analysis of the two-torus via the analytic functions of positive definite type. Section 6 consists of some examples and counterexamples about the analytic functions of positive definite type.

The paper is based on the author's dissertation written under the guidance of Professor John L. Kelley of the University of California, Berkeley. The author would like to thank Professor Kelley for help and advice.

1. Reproducing kernels and analytic functions of positive type. We start with the definition of a reproducing kernel. Let 
$H$ be a Hilbert space of functions on a set $X$ with the inner product (, ). In Theorem 1.2 we present a few facts of a theory of $H$ due essentially to E. H. Moore and N. Aronszajn. See [1] and [2].

Definition 1.1. A reproducing kernel for $H$ is a complex valued function $K$ on $X \times X$ such that:

(i) For all $y \in X, K_{y} \in H$ where $K_{y}(x)=K(x, y)$.

(ii) If $g \in H$ then for all $y \in X,\left(g, K_{y}\right)=g(y)$, and

(iii) The linear span of the set $\left\{K_{y}\right\}_{y \in X}$ of functions is dense in $H$.

Note that if $K$ is a reproducing kernel then the map $g \mapsto g(y)=$ $\left(g, K_{y}\right)$ is a bounded linear functional on $H$; i.e., evaluation at a point is continuous. Also if $H$ is a pre-Hilbert space of funtions on a set $X$ with continuous evaluation then the completion $\bar{H}$ of $H$ can be realized as a space of functions on $X$ by setting $h(x)=\left(h, K_{x}\right)$ for $x \in X$ and $h \in \bar{H}$ where $K_{x} \in \bar{H}$ is such that $\left(f, K_{x}\right)=f(x)$ for all $f \in H$.

THEOREM 1.2. Let $H$ be a pre-Hilbert space of functions on a set $X$ so that the evaluation map $f \mapsto f(x)$ is continuous for all $x \in X$, let $\bar{H}$ be the completion of $H$. Then the following hold

(i) There is a unique reproducing kernel $K$ for $H$.

(ii) If, for $x \in X, \varepsilon_{x} \in \bar{H}$, is such that $f(x)=\left(f, \varepsilon_{x}\right)$ for all $f \in H$ then $K(x, y)=\left(\varepsilon_{y}, \varepsilon_{x}\right)$.

(iii) If $\left\{b_{i}\right\}_{i \in I}$ is an orthonormal basis for $\bar{H}$, then $K(x, y)=$ $\sum_{i \in I} b_{i}(x) \overline{b_{i}(y)}$, and

(iv) If $H$ is the set of finite formal sums $\sum_{i=0}^{n} a_{i} x_{i}$, where $x_{i} \in X$ and $a_{i}^{\prime}$ s are complex numbers, with an inner product (,) given by $\left(\sum_{i=1}^{n} a_{i} x_{i}, \sum_{j=1}^{m} b_{j} y_{j}\right)=\sum_{i=1}^{n} \sum_{j=1}^{m} a_{i} \bar{b}_{j} K\left(y_{j}, x_{i}\right)$, the $\operatorname{map} \quad \sum_{i=1}^{n} a_{i} x_{i} \mapsto$ $\sum_{i=1}^{n} a_{i} K_{x_{i}}$ of formal sums to members of $H$ is inner product preserving and its image is dense in $\bar{H}$.

See [1] for a proof.

A reproducing kernel is a function of positive type in the following sense.

Definition 1.3. A complex valued function $K$ defined on $X \times X$ is a function of positive type iff $\sum_{i, j=1}^{n} a_{i} \bar{a}_{j} K\left(x_{j}, x_{i}\right) \geqq 0$ for all complex numbers $a_{1}, a_{2}, \cdots, a_{n}$ and all members $x_{1}, x_{2}, \cdots, x_{n}$ of $X$.

Since $\quad \sum_{i} a_{i} K\left(x, x_{i}\right)=\left(\sum_{i} a_{i} K_{x_{i}}, K_{x}\right) \leqq \sqrt{K(x, x)} \sqrt{\sum a_{i} \bar{a}_{j} K\left(x_{j}, x_{i}\right)}$ from the Cauchy inequality, a function of positive type $K$ also has a further property, namely that if $\sum_{i, j=1}^{n} a_{i} \bar{a}_{j} K\left(x_{j}, x_{i}\right)=0$ then $\sum a_{i} K\left(x, x_{i}\right)=0$ for all $x \in X$. Thus it is clear from part (iv) of Theorem 1.2 that a function of positive type is a reproducing kernel. (A function of positive type $K$ is necessarily selfadjoint i.e., $K(x, y)=$ $\overline{K(y, x)}$ for all $x, y$ in $X$.) 
Definition 1.4. For each $s>0, D_{s}\left(\bar{D}_{s}\right)$ is the open (closed) disc of radius $s$ about 0 in the complex plane $\mathscr{C} . A_{s}$ is the space of functions defined and conjugate analytic in $D_{s}$ and $A_{s . s}$ the space of functions defined on $D_{s} \times D_{s}$ which are conjugate analytic in the first variable and analytic in the second variable. $\mathscr{P}$ is the space of polynomials with complex coefficients. If $K$ is a function of positve type and is in $A_{s . s}$ for some $s$ then $K$ is an analytic function of positive type abbreviated a.f.p.t.

Let $K \in A_{s, s}$. The following theorem gives an alternate construction of a Hilbert space which is equivalent to $K$ being a function of positive type.

Theorem 1.5. Let $K \in A_{s, s}$. Then

(i) $K$ is an a.f.p.t. if $1 / 4 \pi^{2} \oint_{C} \oint_{C} K(z, w) \overline{p\left(z^{-1}\right)} p\left(w^{-1}\right) \overline{(d z / z)} \times$ $(d w / w) \geqq 0$ for all $p \in \mathscr{P}$ where the path of integration $C$ is a simple contour in $D_{s}$ such that its inverse winds around $\overline{D_{1 / s}}$ once.

(ii) Let $(H,()$,$) be a pre-Hilbert space of functions analytic in$ a neighborhood of $\overline{D_{1 / s}}$ with the inner product $(p, q)_{K}=1 / 4 \pi^{2}$ $\oint_{C} \oint_{C} K(z, w) p\left(w^{-1}\right) \overline{q\left(z^{-1}\right)(d z / z)(d w / w)}$. Then the map which takes a member $K_{\alpha}$ of $H, \alpha$ a member of $D_{s}$, into the rational function $1 /(1-\alpha z)$ is inner-product preserving.

Proof. Consider $1 / 4 \pi^{2} \oint \oint K(z, w) \overline{p\left(z^{-1}\right)} p\left(w^{-1}\right) \overline{(d z / z)}(d w / w)$ as a limit of Riemann sums $1 / 4 \pi^{2} \sum_{i, j=1}^{n} K\left(z_{j}, z_{i}\right) \overline{p\left(z_{j}^{-1}\right)} p\left(z_{i}^{-1}\right) \overline{\left(z_{j+1}-z_{j} / z_{j}\right)} \times$ $\left(z_{i+1}-z_{2} / z_{i}\right)$. Since $K$ is a function of positive type it is clear that $1 / 4 \pi^{2}$ $\oint \oint K(z, w) \overline{p\left(z^{-1}\right)} p\left(w^{-1}\right) \overline{(d z / z)}(d w / w)$ is positive. Let now $a_{1}, a_{2}, \cdots, a_{n}$ be any complex numbers and let $\alpha_{1}, \cdots, \alpha_{n} \in D_{s}$. Consider the rational function $f$ given by $f(z)=\sum_{i} a_{i}\left(1-\alpha_{i} z\right)^{-1}$. Then $\sum_{i, j=1}^{n} a_{i} \bar{a}_{j} K\left(\alpha_{j}, \alpha_{i}\right)=$ $1 / 4 \pi^{2} \oint_{C_{1}} \oint_{C_{2}} K(z, w) \overline{f\left(z^{-1}\right)} f\left(w^{-1}\right) \overline{(d z / z)}(d w / w)=\lim _{i \rightarrow \infty} 1 / 4 \pi^{2} \oint_{C_{1}} \oint_{C_{2}} K(z, w) \times$ $\overline{p_{\imath}\left(z^{-1}\right)} p_{\imath}\left(w^{-1}\right) \overline{(d z / z)}(d w / w) \geqq 0$ where $p_{\imath}$ is a sequence of polynoimials converging to $f$ uniformly on compact subsets of $D_{1 / s}$ and $C_{1}, C_{2}$ are chosen suitably. Thus (i) is proved the proof of (ii) follows from the observation that the norm in the space of rational functions of the function $z \mapsto \sum_{i=1}^{n} a_{i}\left(1-\alpha_{i} z\right)^{-1}$ is $\sum_{i, j=1}^{n} a_{i} \bar{a}_{j} K\left(\alpha_{j}, \alpha_{i}\right)$ which is the same as the norm of $\sum_{i=1}^{n} a_{i} K_{\alpha_{i}}$ in $H$.

2. The kernel function of cyclic triple. Consider now a Hilbert space $H$ and a bounded linear operator $T$ on $H$. We construct certain functions of positive type associated with $T$ and $H$. Some facts from the functional calculus of $T$ are used. (See [12].) If $b$ is a 
complex valued function analytic in the neighborhood of the spectrum $\sigma(T)$ of $T$ define $b(T)$ as follows.

DEFINITION 2.1. Let $C$ be a contour lying in the domain of analyticity of $b$ which winds around each point of $\sigma(T)$ once. Then $b(T)=1 / 2 \pi i \oint_{C} b(z)(z I-T)^{-1} d z$. It is known that $b \mapsto b(T)$ is a linear and multiplicative homomorphism of the space of functions analytic in a neighborhood of $\sigma(T)$ into the space of bounded linear operators on $H$. (See [12], page 199.)

The following result is probably well-known and will be used later.

THEOREM 2.2. Let $T$ be a bounded linear operator on a Hilbert space $H$ and suppose that there is an analytic function $b$ defined and nonzero on a connected neighborhood of the spectrum $\sigma(T)$ of $T$ such that $b(T)=0$. Then there is a nonzero polynomial $p$ such that $p(T)=0$.

We leave the proof of this fact to the reader. See for example [20].

Now we make the following definition.

Definition 2.3. For $r>0 . \quad B_{r}$ is the space of functions analytic in a neighborhood of the closed disc $\bar{D}_{r}$ with topology given as the inductive limit topology of the spaces $A(U)$ of the functions analytic in $s$ neighborhood $U$ of $\bar{D}_{r}$. (See [14], page 219, problem D.)

It is known that $B_{r}$ is a Montel space. (See [14], page 196, problem $\mathrm{F}(\mathrm{e})$.)

For $r>0$, define $\bar{A}_{r}$ as follows.

DefinItion 2.4. $\bar{A}_{r}$ is the space consisting of functions which are complex conjugates of the functions in $A_{r}$.

Let $E$ be a linear space with a locally convex topology $\tau$ and let $E^{\prime}$ be its dual. Then the strong topology for $E^{\prime}$ is the topology of uniform convergence on $\tau$-bounded sets.

The following result will be important in the sequel.

LeMma 2.5. Let $r>0$. Then $\bar{A}_{1 / r}$ and $B_{r}$ are strong duals for the pairing given by $[f, g]=\oint_{C} f(z) g\left(z^{-1}\right) d z / z$ for $f \in B_{r}$ and $g \in \bar{A}_{1 / r}$ where $C$ is the contour $t \mapsto(r+\varepsilon) e^{i t}, 0 \leqq t \leqq 2 \pi$, for some small $\varepsilon$ depending on $f$ and $g$. 
Proof. We present an outline of the proof and refer to [14] for more information. Since $B_{r}$ is a Montel space it is reflexive ([14], $20 \mathrm{~F}(\mathrm{a}))$ and hence it suffices to show that the dual of $B_{r}$, with strong topology is $\bar{A}_{1 / r}$, for the pairing above.

To show this, let for each $z \in D_{1 / r}, h_{z}$ be the member of $B_{r}$ given by $h_{z}(\zeta)=1 /(1-\zeta z)$ for $\zeta \in \bar{D}_{r}$. For any continuous linear functional $g$ on $B_{r}$ let $i_{0}(g)$ be defined by $i_{0}(g)(z)=g\left(h_{z}\right)$. Then $i_{0}(g)$ is a welldefined function and is a member of $\bar{A}_{1 / r}$. Moreover, $g(f)=1 / 2 \pi i$ $\oint_{C} f(z) i_{0}(g)\left(z^{-1}\right) d z / z=1 / 2 \pi i\left[f, i_{0}(g)\right]$, where $C$ is a contour as in the statement of the proposition $\varepsilon$ being so small that it is contained in the domain of holomorphy of $f$. Conversely any member of $\bar{A}_{1 / r}$ defines a continuous linear functional on $B_{r}$ by the above formula and thus $\bar{A}_{1 / r}$ and $B_{r}^{\prime}$ are algebraically isomorphic.

To complete the proof it remains to show that the topology of u.c.c. on $\bar{A}_{1 / r}$ coincides with the strong topology of $B_{r}^{\prime}$. To prove this first observe that each bounded set of $B_{r}$ is contained in a bounded set of $\bar{A}_{r^{\prime}}$ for some $r^{\prime}>r$ ([14], 17G (6) (iii)). Hence it follows that $B_{r}^{\prime}$ with strong topology is metrizable $([14], 18.4)$. Since each bounded subset of $A_{r}$ is uniformly bounded on every compact subset of $D_{r^{\prime}}$, a Cauchy sequence in the u.c.c. topology of $\overline{A_{1 / r}}$ is also a Cauchy sequence in its strong topology. That a Cauchy sequence in the strong topology of $B_{r}^{\prime}$ is also a Cauchy sequence in its u.c.c. topology follows from the observation that for any compact subset $K$ of $D_{1 / r}$ the family of functions $\left\{h_{z}\right\}_{z \in K}$ is bounded in $B_{r}$ and also the fact that for any $h \in \overline{A_{1 / r}},\left[h_{z}, h\right]=2 \pi i h(z)$ where $z \in K$.

Consider now an operator $T$ on a Hilbert space $H$ and let $e \in H$.

Definition 2.6. A Hilbert triple is a triple (T.H.e) where $H$ is a Hilbert space. $T$ a bounded linear operator on $H$ and $e$ a member of $H$. A Hilbert triple is a cyclic triple if the orbit of $e$ (the linear span of the set $\left\{T^{i} e\right\}_{i=0}^{\circ}$ is dense in $H$. If $r=\|T\|$ the map $\beta_{e}: B_{r} \rightarrow H$ is given by $\beta_{e}(b)=b(T) e$.

The map $\beta_{e}$ depends on $T, H$, and $e$. Note that $\beta_{e}\left(B_{r}\right)$ is dense in $H$ iff $(T, H, e)$ is cyclic.

The following is a consequence of Theorem 2.2.

THEOREM 2.7. If $(T, H, e)$ is cyclic then $H$ is infinite dimensional if and only if $\beta_{e}$ is injective.

Proof. If $H$ is finite dimensional then it follows from the CaleyHamilton theorem of linear algebra that $p(T)=0$ for some $p \in \mathscr{P}$ ([4], page 320) and thus $\beta_{e}$ is not injective. Since $(T, H, e)$ is cyclic the converse is the statement of Theorem 2.2. 
Now we establish commutativity of certain maps and construct a reproducing kernel which is an a.f.p.t. The reason for this round about construction will be clear from Theorem 4.3.

Definition 2.8. Multiplication by $z, M_{z}: B_{r} \rightarrow B_{r}$ is the operator given by $M_{z} f(\zeta)=\zeta f(\zeta)$ for $\zeta \in \operatorname{Domain}(f)$.

The following lemma is an easy consequence of the functional calculus.

LEMmA 2.9. $\beta_{e}$ and $M_{z}$ are continuous linear maps such that $\beta_{e}(1)=e$ and $T \cdot \beta_{e}=\beta_{e} \cdot M_{z}$.

Definition 2.10. $\phi: H \rightarrow H^{\prime}$ is defined by $\phi(x)(y)=(y, x)$ for all $x, y H$.

$H^{\prime}$ is conjugate linearly isomorphic to $H$ via the map $\phi$ and $\phi \cdot T^{*}=T^{\prime} \cdot \phi$ where $T^{*}$ is the Hilbert space adjoint of $T$ on $H$ and $T^{\prime}$ is the Banach space dual of $T$. Also from $T \cdot \beta_{e}=\beta_{e} \cdot M_{z}$ we obtain $\beta_{e}^{\prime} \cdot T^{\prime}=M_{z}^{\prime} \cdot \beta_{e}^{\prime}$ where $\beta_{e}^{\prime}, M_{z}^{\prime}$, etc. are Banach space duals of $\beta_{e}, M_{z}$, etc. We know from Lemma 2.5 that $B_{r}^{\prime}$ can be identified with $A_{1 / r}$ via the map $i_{0}$.

Definition 2.11. The map $\alpha_{e}: H \rightarrow A_{1 / r}$ is the composition of the maps $\phi, \beta_{e}^{\prime}, i_{0}$ and - as given in the diagram

$$
H \stackrel{\varphi}{\longrightarrow} H^{\prime} \stackrel{\beta_{e}^{\prime}}{\longrightarrow} B_{r}^{\prime} \stackrel{i_{0}}{\longrightarrow} A_{1 / r} \stackrel{\longrightarrow}{\longrightarrow} A_{1 / r}
$$

where - takes a function into its complex conjugate.

From Lemma 2.9 and the discussion following it we see that there is a map $S^{*}: A_{1 / r} \rightarrow A_{1 / r}$ so that $\alpha_{e} \cdot T^{*}=S^{*} \cdot \alpha_{e}$ where $\alpha_{e}$ is as above.

THEOREM 2.12. Let $(T, H, e)$ be a Hilbert triple with $\|T\|=r$. Then $S^{*} \alpha_{e}=\alpha_{e} \cdot T^{*}$ where $\alpha_{e}$ is such that $\alpha_{e}(x)(z)=\left(x,(1-z T)^{-1} e\right)$ for all $z \in D_{1 / r}$ and all $x \in H$, and $S^{*} f(z)=f(z)-f(0) / z$. Moreover, $\alpha_{e}$ is injective iff $(T, H, e)$ is cyclic and in that case, if $\operatorname{dim} H=\infty$, the range of $\alpha_{e}$ is dense in $A_{1 / r}$.

Proof. We show that $\alpha_{e}$ is such that $\alpha_{e}(x)(z)=\left(x,(1-z T)^{-1} e\right)$ for all $z \in D_{1 / r}$ and $S^{*} f(z)=f(z)-f(0) / z$, because if this were so then $\alpha_{e}\left(T^{*} x\right)(z)=\left(T^{*} x,(1-z T)^{-1} e\right)=\left(x,(1-z T)^{-1} T e\right)=S^{*}\left(\alpha_{e}(x)\right)(z)$ and hence $S^{*} \cdot \alpha_{e}=\alpha_{e} \cdot T^{*}$. Now $-0 i_{0} 0 \beta^{\prime} 0 \phi(x)(z)=\overline{\beta^{\prime} 0 \phi(x)\left(h_{z}\right)}=(x)\left(\beta_{e}\left(h_{z}\right)\right)=$ $\overline{\left((1-z T)^{-1} e, x\right)}=\left(x,(1-z T)^{-1} e\right)$.

Now we prove the rest of the theorem. Since $\alpha_{e}(x)=0$ iff $(x,(1-$ $\left.z T)^{-1} e\right)=0$ for all $z \in D_{1 / r}$ it follows that $\alpha_{e}(x)=0$ iff $(x, p(T) e)=0$ for all $p \in \mathscr{P}$. If $e$ is cyclic then this holds iff $x=0$. The statement 
that if $e$ is cyclic and if $\operatorname{dim} H=\infty$ then the range of $\alpha_{e}$ is dense in $A_{1 / r}$ is a mere dualisation of the statement in Theorem 2.2.

Note that in general $\alpha_{e}$ is injective on the orbit of $e$ and zero on its orthogonal complement. If $e$ is cyclic then we can assign an inner product ()$_{\alpha_{e}}$ on $\alpha_{e}[H]$ by $\left(\alpha_{e}(x), \alpha_{e}(y)\right)_{\alpha_{e}}=(x, y)$. This inner product makes $T^{*}$ on $H$ unitarily equivalent to $S^{*}$ on $\alpha_{e}[H]$. Moreover, and this is the point of the construction, $\alpha_{e}[H]$ has a reproducing kernel.

THEOREM 2.13. Let $(T, H, e)$ be cyclic. Then for each $z \in D_{1 / r}$ the evaluation $\operatorname{map} \alpha_{e}(x) \mapsto \alpha_{e}(x)(z)$ defined on $\alpha_{e}[H]$ is continuous and $\alpha_{e}(x)(z)=\left(\alpha_{e}(x), \varepsilon_{z}\right)$ where $\varepsilon_{z}=\alpha_{e}\left((1-z T)^{-1} e\right)$. Consequently $\alpha_{e}[H]$ has a reproducing kernel $K$ given explicitly as $K(z, w)=((1-$ $\left.w T)^{-1} e,(1-z T)^{-1} e\right)=\sum_{m, n=0}^{\infty}\left(T^{n} e, T^{m} e\right) \bar{z}^{m} w^{n}$.

Proof. Since $\alpha_{e}(x)(z)=\left(x,(1-z T)\left({ }^{-1} e\right)=\left(\alpha_{e}(x), \alpha_{e}\left((1-z T)^{-1} e\right)\right)_{\alpha_{e}}\right.$ for all $\alpha_{e}(x) \in \alpha_{e}[H]$, we see that the evaluation at $z$ is explicitly given by inner product with a member of $\alpha_{e}[H]$ and is hence continuous. Furthermore, the particular member of $\alpha_{e}[H]$ corresponding to evaluation at $z$ is $\alpha_{e}\left((1-z T)^{-1}\right)$ and hence $\varepsilon_{z}=\alpha_{e}\left((1-z T)^{-1} e\right)$. Now in view of Theorem 1.2 it follows that $\alpha_{e}[H)$ has a reproducing kernel given explicitly as $K(z, w)=\left(\alpha_{e}\left((1-w T)^{-1} e\right), \alpha_{e}\left((1-z T)^{-1} e\right)\right)_{\alpha_{e}}=((1-$ $\left.w T)^{-1} e,(1-z T)^{-1} e\right)$.

Definition 2.14. The kernel function of a cyclic triple $(T, H, e)$ is the reproducing kernel of $\alpha_{e}[H]$. If $(T, H, e)$ is just a Hilbert triple, not necessarily cyclic, then the kernel function for $(T, H, e)$ is defined to be the kernel function for $\left(T_{0}, 0(e), e\right)$ where $0(e)$ is the closed orbit of $e$ and $T_{0}$ is the restriction of $T$ to $0(e)$.

Two cyclic triples $\left(T_{1}, H_{1}, e_{1}\right)$ and $\left(T_{2}, H_{2}, e_{2}\right)$ are defined to be unitarily equivalent, $\left(T_{1}, H_{1}, e_{1}\right) \sim\left(T_{2}, H_{2}, e_{2}\right)$, iff there exists a unitary map $U: H_{1} \rightarrow H_{2}$ with $U \cdot T_{1}=T_{2} \cdot U$ and $U\left(e_{1}\right)=e_{2}$.

Let $(T, H, e)$ be a cyclic triple with kernel function $K$. Consider the Hilbert space $\alpha_{e}[H]$ with the inner product ()$_{\alpha_{e}}$ obtained from $(T, H, e)$. We can construct $\alpha_{e}[H]$ from $K$ in the manner of Theorem 1.2 , since $K$ is the reproducing kernel for $\alpha_{e}[H]$. Since $\alpha_{e}(e)=K_{0}$ we have the following as a corollary to the preceding.

COROLLARY 2.15. Let $(T, H, e)$ be a cyclic triple. Then the kernel function $K$ for $(T, H, e)$ is a complete unitary invariant for $(T, H, e)$, i.e., $\left(T^{*}, H, e\right)$ is unitarily equivalent via $\alpha_{e}$ to $\left(S^{*}, \alpha_{e}[H], K_{0}\right)$.

The above corollary can also be deduced directly. See for example [15]. 
3. The triple of an A.F.P.T. In this section we study the kernel function of a triple more closely. First we mention some properties of a kernel function. Note that if $K$ is the kernel function of $(T, H, e)$ where $\|T\|=r$ then $K$ can be written as $\sum_{m, n=0}^{\infty}\left(T^{n} e, T^{m} e\right) \bar{z}^{m} w^{n}$ for $z, w \in D_{1 / r}$. The boundedness of $T$ is reflected in a special property of $K$. The infinite dimensionality of $H$ is also reflected in another property of $K$. We describe these properties. First the following definitions.

Definition 3.1. The map $\mathscr{S}: A_{s, s} \rightarrow A_{s, s}$ is given by $\mathscr{S}(a)(z, w)=$ $[a(z, w)-a(z, 0)-a(0, w)+a(0,0)] /(\bar{z} w)$ for $z, w \in D_{s}$. A member $K$ of $A_{s, s}$ which is an a.f.p.t. is an analytic function of positive definite type (abbreviated a.f.p.d.t.) iff there is a positive real $r$ so that $r^{2} K-\mathscr{S}(K)$ is also an a.f.p.t. We write $\rho(K)$ for the least such $r$. $K \in A_{s, s}$ is a degenerate a.f.p.t. iff $K$ is an f.p.t. and there is a polynomial $p$ so that $\oint_{C} \oint_{C} K(z, w) \overline{p\left(z^{-1}\right)} p\left(w^{-1}\right) \overline{(d z / z)}(d w / w)=(p, p)=0$, where $C$ is such that its inverse contains $\overline{D_{1 / s}}$ and is preferably a circle with center 0 .

It follows from Theorem 1.5 that if ' $a$ ' is a function of positive type so is $\mathscr{S}(a)$, since $\oint_{C} \oint_{C} \mathscr{S}(a)(z, w) \overline{p\left(z^{-1}\right)} p\left(w^{-1}\right) \overline{(d z / z)}(d w / w)=$ $\oint_{C} \oint_{C} a(z, w) p_{1}\left(z^{-1}\right) p_{1}\left(w^{-1}\right) \overline{(d z / z)}(d w / w)$, where $p_{1}$ is the polynomial such that $p_{1}(z)=z p(z)$. As shown by the following result the kernel function of a Hilbert triple is always an a.f.p.d.t.

Proposition 3.2. If $K$ is the kernel function for the triple $(T, H, e)$ then $K$ is an a.f.p.d.t. and $\mathscr{S}(K)$ is the kernel function for the triple $(T, H, T e)$. Moreover, if $(T, H, e)$ is cyclic, then $=\rho(K)=$ $\|T\|$ and $K$ is nondegenerate iff $\operatorname{dim} H=\infty$.

We omit the straightforward proof of this proposition.

Recall that if $(T, H, e)$ is a cyclic triple then we have seen in $\S 2$ (Theorem 2.15) that $\left(T^{*}, H, e\right)$ is unitarily equivalent via $\alpha_{e}$ to $\left(S^{*}, \alpha_{e}[H], K_{0}\right)$ and that $\alpha_{e}[H]$ with ()$_{\alpha_{e}}$ is a space of functions with continuous evaluations at points, thus the space $\alpha_{e}[H]$ and in fact the triple $\left(T^{*}, H, e\right)$ and hence $(T, H, e)$, is determined by the kernel function $K$. We want to describe explicitly in two different ways corresponding to the two Theorems 1.2 and 1.5 of $\S 1$, the construction of $(T, H, e)$ from $K$.

First it follows from $\alpha_{e} \cdot T^{*}=S^{*} \cdot \alpha_{e}$ and the fact that $\alpha_{e}^{*}=\alpha_{e}^{-1}$ that $\alpha_{e} \cdot T=S \cdot \alpha_{e}$ where $S$ is the adjoint of $S^{*}$ relative to the inner product ( $)_{\alpha_{e}}$ of $\alpha_{e}[H]$. We construct $\alpha_{e}[H]$ in terms of the kernel $K$ of $(T, H, e)$. We make the construction for an arbitrary a.f.p.t. 
$K \in A_{s, s}$. A formal for $S$ is given in Proposition 3.4. First a definition.

Definition 3.3. For $K$ an a.f.p.t., $K \in A_{r, r}$, let $H^{K}$ be the completion of the space of functions which are finite linear combinations $\sum_{i} a_{i} K_{\alpha_{i}}$ for $a_{i} \in \mathscr{C}$ and $\alpha_{i} \in D_{r}$ with respect to the inner product $\left(\sum_{i} a_{i} K_{\alpha_{i}}, \sum_{j} b_{j} K_{\beta_{j}}\right)^{k}=\sum_{i, j} a_{i} \bar{b}_{j} K\left(\beta_{j}, \alpha_{i}\right)$.

We have the following result.

Proposition 3.4. Let $K \in A_{r, r}$ be an a.f.p.t. Then $H^{K} \subset A_{r}$. If $K$ is the kernel function for a cyclic triple $(T, H, e)$ then $\alpha_{e}[H]=H^{K}$ and the adjoint $S$ of $S^{*}: H^{K} \rightarrow H^{K}$ is given by $S\left(K_{w}\right)=K_{w}-K_{0} / w$ for $w \neq 0$ and $S\left(K_{0}\right)=d K /\left.d w\right|_{w=0}$.

Proof. The fact that $H^{K}$ consists of functions is known from $\S 1$. Let $f \in H^{K}$ and let $f_{n}=\sum_{i} a_{i}^{n} K_{\alpha_{i}^{n}}$ be a sequence of finite sums converging to $f$. Then since $\left|\sum a_{i} K_{\alpha_{i}}(z)\right|^{2} \leqq K(z, z)\left\|a_{i} K_{\alpha_{i}}\right\|^{2}$ it follows that the sequence $f_{n}$ of conjugate analytic functions is a Cauchy sequences in the topology of uniform convergence on compact sets of $D_{r}$. Hence the limit function $f$ is also conjugate analytic. Thus $H^{K} \subset A_{r}$.

Since, if $K$ is the kernel function for $(T, H, e)$ then $K(z, w)=$ $\left((1-w T)^{-1} e,(1-z T)^{-1} e\right), \alpha_{e}[H]=H^{K}$. Since $e$ is cyclic the adjoint $S$ of $S^{*}$ is given by

$$
\begin{aligned}
& S\left(K_{w}\right)(z)=\left((1-w T)^{-1} T e,(1-z T)^{-1} e\right) \\
= & \left(\frac{(1=w T)^{-1} e-e}{w},(1-z T)^{-1} e\right)=\frac{K_{w}-K_{0}}{w}(z)
\end{aligned}
$$

for $w \neq 0$ and $S\left(K_{0}\right)(z)=\left(T e,(1-z T)^{-1} e\right)=d K /\left.d w\right|_{w=0}(z)$.

COROLlaRY 3.5. If $K$ is the kernal function of the cyclic triple $(T, H, e)$ then $(T, H, e)$ is unitarily equivalent via $\alpha_{e}$ to $\left(S, H^{K}, K_{0}\right)$.

Also this,

COROLLARY 3.6. $\left(S, H^{K}, K_{0}\right)$ is a cyclic triple with kernel function $K$.

Now we make the second construction corresponding to Theorem 1.5 of $\S 1$. First a definition.

Definition 3.7. If $K \in A_{1 / r, 1 / r}$ and $K$ is an a.f.p.t., $H_{K}$ is the completion of the space $B_{r}$ of functions analytic in a neighborhood of $\overline{D_{r}}$ with respect to ()$_{K}$ given by $\left(b_{1}, b_{2}\right)=1 / 4 \pi^{2} \oint_{C_{1}} \oint_{C_{2}} K(z, w) \times$ $b_{1}\left(w^{-1}\right) \overline{b_{2}\left(z^{-1}\right)(d z / z)}(d w / w)$, where $b_{1}, b_{2}$ are members of $B_{r}$ and where 
$C_{i}$ is a contour such that its inverse is rectifiable in the complex plane, contains $\bar{D}_{r}$ and has winding number 1 about $\bar{D}_{r}$ and is contained in the domain of holomorphy of $b_{i}$. If multiplication by $z, M_{z}$ is bounded on $B_{r}$ relative to ( $)_{K}$ then $M_{z}^{\wedge}$ is its bounded extension to $H_{K}$.

$H_{K}$ is well-defined despite the arbitrariness of the contours and $r$. This is so because of Cauchy's theorem. Also note that $H_{K}$ does not consist of functions in general (see counterexample $1, \S 6$ ).

Proposition 3.8. Let $K$ be an a.f.p.t. $\in A_{1 / r, 1 / r}$. Then

(i) $M_{z}$ on $B_{r}$ has bounded extension $M_{z}^{\wedge}$ to $H_{K}$ iff $K$ is an a.f.p.d.t.

(ii) If $K$ is an a.f.p.d.t. then $K$ is the kernel function of $\left(M_{z}^{\wedge}, H_{K}, 1\right)$.

(iii) If $K$ is the kernel function of a cyclic triple $(T, H, e)$ then $\left(M_{z}^{\wedge}, H_{K}, 1\right)$ is unitarily equivalent to $(T, H, e)$ under the map $p \mapsto$ $p(T) e$ for all polynomials $p$.

Proof.

(i ) $K$ is an a.f.p.d.t. iff there is $t \geqq 0$ so that $t^{2} K-\mathscr{S}(K)$ is of positive type. However, $\oint \oint \mathscr{S}(K)(z, w) \overline{a\left(z^{-1}\right)} a\left(w^{-1}\right) \overline{(d z / z)}(d w / w)=$ $\left.\oint \oint K(z, w) \overline{\left(M_{z} a\right)\left(z^{-1}\right)}\left(M_{w} a\right)\left(w^{-1}\right) \overline{(d z / z}\right)(d w / w), a \in B_{r}$. Hence $t^{2} K-\mathscr{S}(K)$ is a function of positive definite type iff $t \geqq\left\|M_{z}^{\wedge}\right\|, B_{r}$ being dense in $H_{K}$. To prove

(ii) note that if $\lambda \in D_{1 / r}$ then the function $R_{\lambda}: z \rightarrow(1-\lambda z)^{-1} \in B_{r}$ and $\left(R_{\lambda}, R_{\mu}\right)=1 / 4 \pi^{2} \oint_{C_{1}} \oint_{C_{2}} K(z, w) 1 /\left(\left(1-\lambda w^{-1}\right) \overline{\left.\left(1-\mu z^{-1}\right)\right)(d z / z)}(d w / w)\right.$ where $C_{1}, C_{2}$ are contours specified as in Definition 3.7. It follows from the Cauchy formula (see [13], page 26) that $\left(R_{\lambda}, R_{\mu}\right)=K(\lambda, \mu)$ and thus (ii) is proved.

The proof of (iii) is straightforward and is left to the reader.

CoROLlary 3.9. A function $K$ is the kernel function for some cyclic triple iff $K$ is an a.f.p.d.t.

\section{Further properties of an A.F.P.T.}

Definition 4.1. The linear map $C^{\prime}: H^{K} \rightarrow H_{K}$ is given on $K_{\alpha}^{\prime} \mathbf{s}$ by $C^{\prime}\left(K_{\alpha}\right)(z)=(1-\alpha z)^{-1}$ and the map $J: H_{K} \rightarrow H^{K}$ is given on polynomials by $J(p)=1 / 2 \pi i \oint_{C} K_{w} p\left(w^{-1}\right) d w / w$ for a simple contour $C$ which lies in the domain of analyticity of the function $w \mapsto K_{w}$ and whose inverse contains $\overline{D_{r}}$ and has winding number 1 about it. 
To be sure we should refer to vector valued integration but here we mean that $J(p)$ is the function so that $J(p)(z)=1 / 2 \pi i \oint_{C} K(z, w) \times$ $p\left(w^{-1}\right) d w / w$. Clearly $J(p)$ is independent of the contour $C$ so long it has the properties given in the definition.

We have the following proposition.

Proposition 4.2. If $(T, H, e)$ is a cyclic triple then $\alpha_{e}$ and $C^{\prime}$ are unitary and the diagram

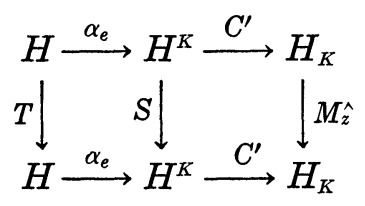

is commutative. Moreover, $C^{\prime-1}=J$ for any a a.f.p.t. $K$ and $C^{\prime}$ is unitary for any a.f.p.t.

Proof. We know that $\alpha_{e}$ is unitary. That $C^{\prime}$ is unitary for any a.f.p.t. $K$ is a consequence of Theorem 1.5. Since $\alpha_{e}[H]=H^{K}$ we know that the diagram

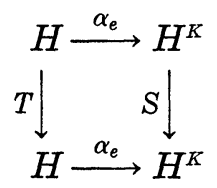

is commutative. To prove that the rest of the diagram is also commutative it suffices to prove that $C^{\prime} \cdot S=M_{z}$. $C^{\prime}$ on elements of the type $K_{\alpha}$ of $H^{K}$. However, this is true since $C^{\prime} \cdot S\left(K_{\alpha}\right)(z)=C^{\prime}$.

$$
\frac{K_{\alpha}-K_{0}}{\alpha}(z)=\frac{(1-\alpha z)^{-1}-1}{\alpha}=z(1-\alpha z)^{-1}=M_{z} \cdot C^{\prime}(K)(z) .
$$

Now we prove that $C^{\prime-1}=J$ for any a.f.p.t. $K$. To do so it suffices to consider elements of the form $C^{\prime}\left(K_{\alpha}\right)$ of $H_{K}$. For such an element

$$
J \cdot C^{\prime}\left(K_{\alpha}\right)=\frac{1}{2 \pi i} \oint_{C} K_{w}\left(1-\alpha w^{-1}\right)(d w / w)=\frac{1}{2 \pi i} \oint_{C} \frac{K_{w}}{w-\alpha} d w=K_{\alpha} .
$$

Now let $H$ be the closure of $B_{r}$ under an inner product. Then we have the

THEOREM 4.3. $H=H_{K}$ for some a.f.p.t. $K \in A_{1 / r},{ }_{1 / r}$ iff $H^{*}=H^{K}$.

Proof. If $H=H_{K}$ then the inclusion of $B_{r}$ in $H$ is continuous 
where $B_{r}$ is taken with its u.c.c. topology. Hence the dual of $H$ can be identified with $H^{K}$ as in Proposition 4.2. Conversely if $H^{*}=H^{K}$ for some a.f.p.t. $K$ in $A_{1 / r}, 1 / r$, then the dual can be identified with $H$ is the same as $H_{K} \subset B_{r}$ via the map $C^{\prime}$.

The following two theorems give characteristics of an a.f.p.d.t. supplementing Definition 3.1 and furnishing a connection between an a.f.p.d.t. and an invariant subspace.

THEOREM 4.4. Let $K \in A_{r, r}$ be an a.f.p.t. Then $K$ is an a.f.p.d.t. if and only if $H^{K}$ is $S^{*}$-invariant.

The above theorem is an immediate consequence, of the fact that the inclusion of $H^{K}$ in $A_{r}$ is continuous, and the closed graph theorem. We leave the proof to the reader.

Let $(T, H, e)$ be a cyclic triple. We prove another proposition which relates invariant subspaces of $T$ in $H$ to functions of positive definite type arising from the kernel function of $(T, H, e)$ and the projection corresponding to the invariant subspace.

THeOREM 4.5. Let $(T, H, e)$ be a cyclic triple. Then $P$ is an orthogonal projection so that $P(H)$ is invariant under $T$ if and only if the function $K^{\prime}$ defined by $K^{\prime}(z, w)=\left((I-P)(1-w T)^{-1} e,(I-P) \times\right.$ $\left.(1-z T)^{-1} e\right)$ is an a.f.p.d.t.

We have to show that $P(H)$ is invariant under $T$ if and only if the operator $(I-P) x \mapsto(I-P) T x$ is bounded. We prove this fact as a consequence of a more general lemma.

Lemma 4.6. Let $(T, H, e)$ be a Hilbert triple and $\widetilde{H}$ a first countable Hausdorff linear topological space, $\widetilde{T}$ a continuous linear operator defined on $\widetilde{H}$ and $\widetilde{e}$ a member of $\tilde{H}$. Let $H_{\tilde{e}}=\{x \in H$; there is a sequence $\left\{p_{i}(T) e\right\}_{i} \rightarrow x$ and $\left\{p_{i}(\widetilde{T}) \tilde{e}\right\}_{i} \rightarrow 0$ where each $p_{i} \in P, i=$ $1,2, \cdots\}$. Then $H_{\tilde{e}}$ is a closed invariant subspace for $T$.

Proof. $H_{\tilde{e}}$ is clearly a linear subspace of $H$ invariant under $T$. The whole difficulty lies in showing that it is closed. This is also quite straightforward and we do it as follows. Let $\left\{p_{i}^{m}(T) e\right\}_{i}$ be a sequence converging to $x_{m}$ and let also the $\left\{x_{m}\right\}_{m}$ converge to $x$. Also let $\left\{p_{i}^{m}(\widetilde{T}) \widetilde{e}\right\}_{i}$ converge to 0 for each $m$. Let $B\left(x, 2^{-n}\right)\left(\bar{B}\left(x, 2^{-n}\right)\right)$ be the open (closed) ball of radius $2^{-n}$ with center at $x$ and let $\left\{n_{i}\right\}_{i}$ be the sequence for which $\left\{x_{m}\right\}_{m} \cap B\left(x, 2^{-n_{i}}\right)-\bar{B}\left(x, 2^{-n_{i}-1}\right) \neq \varnothing$. Such a sequence $\left\{n_{i}\right\}_{i} \rightarrow \infty$ exists since $\left\{x_{m}\right\}_{m} \rightarrow x$. For each $n_{i}$ choose an $x_{n_{i}} \in B\left(x, 2^{-n_{i}}\right)-\bar{B}\left(x, 2^{-n_{i}-1}\right)$. Suppose also that $\left\{U_{n_{i}}\right\}_{i}$ is a countable neighborhood basis at 0 for $\widetilde{H}$. For each $n_{i}$ choose a $p_{j\left(n_{i}\right)}^{n_{i}}$ so that $p_{j\left(n_{i}\right)}^{n_{i}}(\widetilde{T}) \tilde{e} \in U_{n_{i}}$ and $p_{j\left(n_{i}\right)}^{n_{i}}(T) e$ is in $B\left(x, z^{-n_{i}}\right)-\bar{B}\left(x, 2^{-n_{i}-1}\right)$. It is possible 
to do so since $\left\{p_{j}^{n_{i}}(T) e\right\}_{j} \rightarrow x_{n_{i}}$ and $\left\{p_{j}^{n_{i}}(\widetilde{T}) \widetilde{e}\right\}_{j} \rightarrow 0$ for each $n_{i}$ by supposition.

Now the sequence $\left\{p_{j\left(n_{i}\right)}^{n_{i}}(\widetilde{T}) \widetilde{e}\right\}_{n_{i}}$ has 0 as a cluster point in $\tilde{H}$ so there is a proper infinite subsequence of this sequence which tends to 0 . Since $\left\{p_{j\left(n_{i}\right)}^{n_{i}}(T) e\right\}_{n_{i}} \rightarrow x$ in that subsequence if we replece $T$ and $e$ by $T$ and $e$ it tends to $x$. Thus $H_{\tilde{e}}$ is closed.

The proof of this lemma can perhaps be simplified by construction of a bounded intertwining operator from $H$ to $\widetilde{H}$ whose kerne] coincides with the subspace given by the limits of $\left\{p_{i}(T) e\right\}_{i}$ as above.

Now we complete the proof of Theorem 4.5 as follows.

Proof of Theorem 4.5. Suppose that $P(H)$ is invariant for $T$. We know that $\|p\|_{H_{K^{\prime}}}=\|(I-P) p(T) e\|_{H}$. Thus $\left\|M_{z} p\right\|_{H_{K^{\prime}}}=$ $\|(I-P) T p(T) e\|_{H}=\|(I-P) T P p(T) e+(I-P) T(I-P) p(T) e\|_{H}=$ $\|(I-P) T(I-P) p(T) e\|_{H} \leqq\|T(I-P) p(T) e\|_{H} \leqq M\|(I-P) p(T) e\|_{H}=$ $M\|p\|_{H_{K}}$, where $M$ is the norm of $T$, and the suffix indicates the space in which norm is to be taken. It follows that $M_{z}$ is bounded on $H_{K^{\prime}}$ and hence $K^{\prime}$ is an a.f.p.d.t.

In order to prove the converse we use Lemma 4.6 and put for $\widetilde{H}, \widetilde{T}, \widetilde{e} H_{K^{\prime}}, M_{z}^{\wedge}$ and 1 respectively.

We conclude by characterizing interlacing maps of cyclic triples to another triple in terms of kernel functions as a preparation for $\S 5$.

THEOREM 4.7. Let $\left(T_{1}, H_{1}, e_{1}\right)$ be a cyclic triple and let $\left(T_{2}, H_{2}, e_{2}\right)$ be another triple and $\phi_{12}$ the unique map given by $\phi_{12}\left(p\left(T_{1}\right) e_{1}\right)=p\left(T_{2}\right) e_{2}$ for all $p \in \mathscr{P}$. Then $\phi_{12}$ extends to a unique bounded linear operator $\phi_{12}: H_{1} \rightarrow H_{2}$ iff there exists a real number $r$ so that $r^{2} K_{1}-K_{2}$ is an a.f.p.t. where $K_{1}$ and $K_{2}$ are the kernel functions of the triples $\left(T_{1}, H_{1}, e_{1}\right)$ and $\left(T_{2}, H_{2}, e_{2}\right)$ respectively.

5. A category of triples and harnomic analysis. Let $\mathscr{C}$ be the class of all triples $(T, H, e)$ so that $T$ is a proper contraction. Then $\mathscr{C}$ becomes a category if we define morphism between triples $\left(T_{1}, H_{1}, e_{1}\right)$ and $\left(T_{2}, H_{2}, e_{2}\right)$ to be a bounded linear map $\phi_{12}: H_{1} \rightarrow H_{2}$ so that $\phi_{12}\left(e_{1}\right)=e_{2}$ and $T_{2} \cdot \phi_{12}=\phi_{12} \cdot T_{1}$. We discuss some elementary facts about this category. See [16] for terminology.

Let $H_{1}$ and $H_{2}$ be two Hilbert spaces with linear operators $T_{1}$ and $T_{2}$. Then $H_{1} \oplus H_{2}$ and $T_{1} \oplus H_{2}$ on $H_{1} \oplus H_{2}$ are defined as usual. (See [17].) It is well-known that if $T_{1}: H_{1} \rightarrow H_{1}$ and $T_{2}: H_{2} \rightarrow H_{2}$ are bounded linear operators then $T_{1} \oplus T_{2}$ is also bounded and infact

$$
\text { \| } T_{1} \oplus T_{2} \| \leqq \max \left(\left\|T_{1}\right\|,\left\|T_{2}\right\|\right) \text {. }
$$

We summarize some properties of this category $\mathscr{C}$ in the following lemma. 
Lemma 5.1. The following hold in the category $\mathscr{C}$ :

(i) A morphism $\phi_{12}:\left(T_{1}, H_{1}, e_{1}\right) \rightarrow\left(T_{2}, H_{2}, e_{2}\right)$ is an epimorphism iff $\phi_{12}\left(H_{1}\right)$ is dense in $H_{2}$.

(ii) If $(T, H, e)$ is cyclic then there is at most one morphism from $(T, H, e)$ to any other object $\left(T_{1}, H_{1}, e_{1}\right)$ of $\mathscr{C}$.

(iii) $(T, H, e)$ is cyclic iff every morphism to it is an epimorphism.

(iv) $\mathscr{C}$ has a terminal object, namely the triple $(0,0,0)$.

(v) The operation $\oplus$ is a sum as well as a product in $\mathscr{C}$.

(vi) $A$ morphism $\phi_{12}:\left(T_{1}, H_{1}, e_{1}\right) \rightarrow\left(T_{2}, H_{2}, e_{2}\right)$ is a monomorphism iff $\operatorname{Ker} \phi_{12}=\{0\}$.

Proof. We prove only (vi) and leave the proof of the rest of the proposition to the reader. If $\phi_{12}: H_{1} \rightarrow H_{2}$ is an injection then clearly $\phi_{12}:\left(T_{1}, H_{1}, e_{1}\right) \rightarrow\left(T_{2}, H_{2}, e_{2}\right)$ is a monomorphism. To prove the converse let $\phi_{12}$ be a monomorphism. Then we have to show that $\operatorname{Ker}\left(\phi_{12}\right)=\{0\} . \quad$ It is easy to see that $\operatorname{Ker}\left(\phi_{12}\right)$ is a closed subspace of $H_{1}$ invariant under $T_{1}$. Consider the triple $\left(T_{1} \oplus T_{1}, H_{1} \oplus \operatorname{Ker}\left(\phi_{12}\right), e_{1} \oplus 0\right)$. Define the morphisms $f$ and $g:\left(T_{1} \oplus T_{1}, H_{1} \oplus \operatorname{Ker}\left(\phi_{12}\right), e_{1} \oplus 0\right) \rightarrow$ $\left(T_{1}, H_{1}, e_{1}\right)$ by setting $f=p_{1}$ and $g=\phi_{11}+\phi_{11}$ where $p_{1}$ is the projection on the first coordinate and $\phi_{11}$ is the identity mapping of $\left(T_{1}, H_{1}, e_{1}\right)$ into $\left(T_{1}, H_{1}, e_{1}\right)$ and $\tilde{\phi}_{11}:\left(T_{1}, \operatorname{Ker}\left(\phi_{12}\right), 0\right) \rightarrow\left(T_{1}, H_{1}, 0\right)$ is the injection of $\operatorname{Ker}\left(\phi_{12}\right)$ into $H_{1}$. Thus $g(x \oplus y)=x+y$. We see now that the morphisms $\phi_{12} g$ and $\phi_{12} f$ are equal. However $f \neq g$. Thus $\phi_{12}$ is not a monomorphism if $\operatorname{Ker}\left(\phi_{12}\right) \neq\{0\}$.

The category $\mathscr{C}$ also admits the usual tensor product operation $\otimes$. $\quad H_{1} \otimes H_{2}$ and its $l^{2}$-completion $H_{1} \hat{\otimes} H_{2}, T_{1} \otimes T_{2}$ and its extension $T_{1} \hat{\otimes} T_{2}$ are defined as usual. It is well-known that if $T_{1}$ and $T_{2}$ are bounded linear operaters then so is $T_{1} \otimes T_{2}$ and in fact $\left\|T_{1} \otimes T_{2}\right\| \leqq$ $\left\|T_{1}\right\|\left\|T_{2}\right\|$. (See [6].)

LEMma 5.2. The operation $\otimes$ is a product in $\mathscr{C}$.

We leave the proof of this statement to the reader. It is easy to check that $\otimes$ is not a sum in $\mathscr{C}$ (see counterexample $2, \S 6$ ).

Unfortunately we do not know an abstract characterization of the category $\mathscr{C}$.

Definition 5.3. An atom in a catogory is an object such that every morphism from it is either zero or a monomorphism.

Theorem 5.4. Let $(T, H, e) \in \mathscr{C}$ be a cyclic triple then $T$ has no proper invariant subspaces in $H$ iff $(T, H, e)$ is an atom in $\mathscr{C}$.

Proof. If $T$ has no proper invariant subspaces then it is clear from (vi) Lemma 5.1 that $(T, H, e)$ is an atom. If $T$ has a proper 
invariant subspace let it be given by the range of an orthogonal projection $P$. Let $(T, H, e)$ be written as $(T, \overline{\{p(T) e \mid p \in \mathscr{P}\}}, e)$ consider also the triple $\left(M_{z}^{\wedge}\{\mathscr{P}\}_{P}, 1\right)$ where the inner product on $\{\mathscr{P}\}_{P}$ is given by $(p, q)=((I-P) p(T) e,(I-P) q(T) e)$. Then $\left(M_{z}^{\wedge},\{\mathscr{P}\}_{P}, 1\right)$ is a cyclic triple (Theorem 4.5). Now the desired morphism $\dot{\phi}_{12}:(T, \overline{\{p(T) e \mid p \in \mathscr{P}\}}, e) \rightarrow\left(M_{z}^{\wedge},\{\mathscr{P}\}_{P}, 1\right)$ is given by $\phi_{12}(p(T) e)=p$.

It is not clear whether the category $\mathscr{C}$ also has an initial object. However, if we adjoin to $\mathscr{C}$ the triple $\left(M_{z}, H^{2}, 1\right)$ where $H^{2}$ is the Hardy space of analytic functions defined on $D_{1}$ whose boundary values are square integrable on the unit circle, and obtain another category $\mathscr{C}^{\prime}$ then $\mathscr{C}^{\prime}$ certainly has an initial object; namely the triple $\left(M_{z}, H^{2}, 1\right)$. This will be given as a corollary in the latter part of this paper.

We now make the following definition and show that the kernel function map $k$ defined as below connects $\mathscr{C}$ and the harmonic analysis of the two-torus.

Definition 5.5. The map $k$ named the kernel function map is the map which assigns to a member $(T, H, e)$ of $\mathscr{C}$ its kernel function.

Now we prove the following properties of $k$ related to the operations $\oplus$ and $\otimes$ of $\mathscr{C}$.

THEOREM 5.6. If $K_{1}$ and $K_{2}$ are the kernel functions for $\left(T_{1}, H_{1}, e_{1}\right)$ and $\left(T_{2}, H_{2}, e_{2}\right)$ respectively, then the kernel functions for $\left(T_{1} \oplus T_{2}, H_{1} \oplus\right.$ $\left.H_{2}, e_{1} \oplus e_{2}\right)$ and $\left(T_{1} \hat{\otimes} T_{2}, H_{1} \hat{\otimes} H_{2}, e_{1} \otimes e_{2}\right)$ are $K_{1}+K_{2}$ and $K_{1} * K_{2}$ respectively where $K_{1} * K_{2}(z, w)=\int_{0}^{2 \pi} \int_{0}^{2 \pi} K\left(z e^{-i \theta}, w e^{-i \xi}\right) K_{2}\left(e^{i \theta}, e^{i \gamma^{\prime}}\right)(d \theta / 2 \pi)(d \psi / 2 \pi)$ and where $d \theta / 2 \pi$ and $d \psi / 2 \pi$ denote the normalized Haar measure of the circle.

Proof. Let $C_{m, n}^{1}$ and $C_{m, n}^{2}$ be the coefficients of $\bar{z}^{m} w^{n}$ in a Taylor expansion of the kernel functions of the triples $\left(T_{1} \oplus T_{2}, H_{1} \oplus H_{2}, e_{1} \oplus e_{2}\right)$ and $\left(T_{1} \otimes T_{2}, \hat{H}_{1} \otimes H_{2}, e_{1} \otimes e_{2}\right)$ respectively.

Then,

$$
\begin{aligned}
C_{m, n}^{1} & =\left(\left(T_{1} \oplus T_{2}\right)^{n}\left(e_{1} \oplus e_{2}\right),\left(T_{1} \oplus T_{2}\right)^{m}\left(e_{1} \oplus e_{2}\right)\right)_{H_{1} \oplus H_{2}} \\
& =\left(T_{1}^{n} \oplus T_{2}^{n}\left(e_{1} \oplus e_{2}\right), T_{1}^{m} \oplus T_{2}^{m}\left(e_{1} \oplus e_{2}\right)\right)_{H_{1} \oplus H_{2}} \\
& =\left(T_{1}^{n} e_{1}, T_{1}^{m} e_{1}\right)_{H_{1}}+\left(T_{2}^{n} e_{2}, T_{2}^{m} e_{2}\right)_{H_{2}}
\end{aligned}
$$

and

$$
\begin{aligned}
C_{m, n}^{2} & =\left(\left(T_{1} \hat{\otimes} T_{2}\right)^{n}\left(e_{1} \otimes e_{2}\right),\left(T_{1} \hat{\otimes} T_{2}\right)^{m}\left(e_{1} \otimes e_{2}\right)\right)_{H_{1} \hat{\otimes}_{H_{2}}} \\
& =\left(T_{1}^{n} \otimes T_{2}^{n}\left(e_{1} \otimes e_{2}\right), T_{1}^{m} \otimes T_{2}^{m}\left(e_{1} \otimes e_{2}\right)\right)_{H_{1} \otimes H_{2}} \\
& =\left(T_{1}^{n} e_{1}, T_{1}^{m} e_{1}\right)_{H_{1}}\left(T_{2}^{n} e_{2}, T_{2}^{m} e_{2}\right)_{H_{2}}
\end{aligned}
$$


and hence $\sum_{m, n=0}^{\infty} C_{m, n}^{1} z^{-m} w^{n}=K_{1}(z, w)+K_{2}(z, w)$. We also observe that since $T_{1}$ and $T_{2}$ are proper contractions $K_{1}$ and $K_{2} \in A_{s, s}$ for some $s>1$ and hence

$$
\begin{aligned}
\sum_{m, n=0}^{\infty} C_{m, n}^{2} z^{-m} w^{n}= & \int_{0}^{2 \pi} \int_{0}^{2 \pi} \sum_{m, n=0}^{\infty}\left(T_{1}^{n} e_{1}, T_{1}^{m} e_{1}\right) \bar{z}^{m} e^{i m \theta} w^{n} e^{-i n \psi} \\
& \times \sum_{m, n=0}^{\infty}\left(T_{2}^{n} e_{2}, T_{2}^{m} e_{2}\right) e^{-i m \theta} e^{i n \psi} d \theta / 2 \pi d \psi / 2 \pi \\
= & \int_{0}^{2 \pi} \int_{0}^{2 \pi} K\left(z e^{-i \theta}, w e^{-i \psi}\right) K\left(e^{i \theta}, e^{i \psi}\right) d \theta / 2 \pi d \psi / 2 \pi .
\end{aligned}
$$

DEFINITION 5.7. The equivalence relation $\simeq$ defined on $\mathscr{C}$ is such that $(T, H, e) \simeq\left(T_{1}, H_{1}, e_{1}\right)$ iff there is a unitary map $\phi: 0(e) \rightarrow$ $0\left(e_{1}\right)$ so that $\phi(e)=e_{1}$ and $\phi \cdot T=T_{1} \cdot \phi$.

Now let us consider the category $\mathscr{C}$ with the equivalence relation $\simeq$ and denote this new object by $\underset{\mathscr{C}}{\simeq}$. Then the operations $\oplus$ and $\otimes$ are defined on $\underset{\mathscr{C}}{\simeq}$ and we have the following corollary.

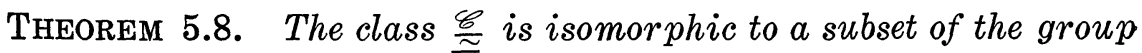
algebra of the torus and so $\underset{i t}{\widetilde{T}}$ is a cancellative abelian semigroup under the operation $\oplus$. It also has no divisors of zero under the operation $\otimes$.

We remark that the set of a.f.p.t.'s is closed under pointwise multiplication. However, the set of a.f.p.d.t.'s is not closed under pointwise multiplication. (See counterexample 3.)

6. Some examples and counterexamples.

EXAMPLES.

1. Consider $\left(M_{z}, H^{2}, 1\right)$. Then $\left(M_{z}, H^{2}, 1\right)$ is a cyclic triple and its kernel function $K$ is given by

$$
K(z, w)=\sum_{m, n=0}^{\infty}\left(z^{n}, z^{m}\right) \bar{z}^{m} w^{n}=(1-\bar{z} w)^{-1} .
$$

If $q$ is an inner function then $k\left(M_{z}, H^{2}, 1\right)=k\left(M_{z}, H^{2}, q\right)$.

2. The triple $\left(V, L^{2}[0,1], 1\right)$, where $V$ is the Volterra operator on the Hilbert space of square integrable real-valued functions on the closed interval $[0,1]$ with Lebesgue measure, is also a cyclic triple. Its kernel function $K$ is given by

$$
\begin{aligned}
K(z, w) & =\sum_{m, n=0}^{\infty}\left(V^{n}(1), V^{m}(1)\right) z^{-m} w^{n} \\
& =\int_{0}^{1} e^{x}(\bar{z}+w) d x=\frac{e^{\bar{z}+w}-1}{\bar{z}+w} .
\end{aligned}
$$


3. The kernel function of the triple $\left(M_{\alpha}, \mathscr{C}, 1\right)$ is the function $1 \sqrt{(1-\alpha z)^{-1}}(1-\alpha w)^{-1}$. This is a special case of an example to be given in the latter part of this paper.

\section{Counterexamples.}

1. This counterexample shows that in general $H_{K}$ is not a space of functions. We claim that the following holds.

Let $\left(M_{z}^{\wedge}, H_{K}, 1\right)$ be a canonical triple with

$$
K(z, w)=\sum_{n=0}^{\infty} e^{2\left(-e^{n}+1\right)} \bar{z}^{n} w^{n} .
$$

Let $M(X)$ denote the space of functions (measurable or otherwise) for a Borel field $(X, A, \mu)$ finite valued except for a set of measure zero, and let it be given the topology of pointwise convergence. Then the identity map $i$ of the polynomials contained in $H$ to the polynomials contained in $M(X)$ if injective is not continuous. This is seen as follows.

First the space $M(X)$ is complete. Second the sequence of functions $\left\{f_{n}\right\}_{n}$ where $f_{n}(z)=e^{e^{n}-1} z^{n}$ is a complete orthonormal sequence in $H_{K}$. Hence the sequence of polynomials $\left\{p_{n}\right\}_{n \in N}$ given by $p_{n}(z)=$ $\sum_{i=0}^{n} 2^{-i} e^{e^{i}-1} z^{i}$ converges to a member $h$ of $H_{K}$. Now let $X$ be any subset of the complex plane $\ell$. Since $i$ is injective $X \neq\{0\}$. It suffices to show that the sequence $p_{n}(z)_{n}$ does not converge for any $z \in \ell, z \neq 0$. Let $z=r e^{i \theta}$. Then $\left|p_{n}(z)-p_{n-1}(z)\right|=e^{e^{n-1}}(r / 2)^{n}=$ $e^{e^{n}-1+n \log r / 2}$, which tends to $\infty$ if $r \neq 0$. Hence the sequence $\left\{p_{n}(z)\right\}_{n}$ is divergent unless $z=0$.

2. If $(T, H, e)$ is a cyclic triple then the subspace $\{p(T) e \mid p \in \mathscr{P}\}$ can be given the structure of an abelian ring if we define $p(T) e \cdot q(T) e=p q(T) e$. However, this operation is not continuous and does not extend to all of $H$. Take for $(T, H, e)$ the triple $\left(M_{z}, H^{2}, 1\right)$. Consider the convergent sequence $\left\{p_{n}\right\}_{n}$ given by $p_{n}(z)=$ $\sum_{m=1}^{n} z^{m} / \mathrm{m}^{3 / 5}$. The limit is of course in $H^{2}$. However, a simple calculation shows that $p_{n}^{2}$ does not converge in $H^{2}$. We leave the calculation to the reader. (To see how it relates to $\otimes$ not being a sum in $\mathscr{C}$ take for $j_{1}:(T, H, e) \rightarrow(T \widehat{\otimes} T, H \widehat{\otimes} H, e \otimes e)$ the map $f(T) e \mapsto$ $f(T) e \otimes e$ and for $j_{2}:(T, H, e) \rightarrow(T \widehat{\otimes} T, H \widehat{\otimes} H, e \otimes e)$ the map $g(T) e \rightarrow$ $e \otimes g(T) e$ and let $\left(T_{3}, H_{3}, e_{3}\right)$ be the triple $(T, H, e)$ with $\phi_{13}$ and $\phi_{23}$ as the identity map $(f(T) e \mapsto f(T) e)$ then there is no map from $\phi:(T \widehat{\otimes}$ $T, H \hat{\otimes} H, e \otimes e) \rightarrow(T, H, e)$ so that $\phi \circ j_{1}=\phi_{13}$ and $\phi \circ j_{2}=\phi_{23}$.)

3. We show now that every a.f.p.t. is not an a.f.p.d.t. as well as the pointwise product of two a.f.p.d.t.'s is not necessarily an a.f.p.d.t. The kernel function of the triple $(1, \mathscr{C}, 1)$ is the function 
$K(z, w)=(1-\bar{z})^{-1}(1-w)^{-1}$. This is an a.f.p.d.t. Its square the function $K_{1}(z, w)=(1-\bar{z})^{-2}(1-w)^{-2}$ is obviously an a.f.p.t. But it is not an a.f.p.d.t. as $H_{K}$ is one dimensional and the a.f.p.d.t. corresponding to a triple $\left(M_{\alpha}, \mathscr{C}, \beta\right)$ is the function $\beta^{2}(1-\bar{\alpha} \bar{z})^{-1}(1-\alpha w)^{-1}$.

\section{REFERENCES}

1. N. Aronszajn, Theory of reproducing kernels, Trans. Amer. Math. Soc., 68 (1950), 337-404.

2. S. Bergman, The Kernel Function and Conformal Mapping, American Mathematical Society Surveys Number V, New York, 1950.

3. A. Beurling, On two problems concerning linear transformations in Hilbert space, Acta. Math., 81 (1949), 239-255.

4. G. Birkhoff and S. MacLane, A Survey of Modern Algebra, Revised edition, Macmillan Co., New York, 1963.

5. S. Bochner and W. T. Martin, Several Complex Variables, Fourth printing, Princeton University Press, 1964.

6. J. Dixmier, Les Algebres d' Operateurs danse Lé Espace Hilbertian, (Algebres de Von Neumann), Gauthier-Villaries, 55 Quai des Grandes Augustins, 1957.

7. N. Dunford and J. Schwartz, Linear Operators, Part II, Interscience, Wiley and Sons, New York, Third printing, March, 1967.

8. I. C. Gohberg and M. G. Krein, Theory and Applications of Volterra Operators in Hilbert Space, Translation from the Russian by A. Feinstein, Amer. Math. Soc., Providence, R. I., 1970.

9. P. R. Halmos, Finite Dimensional Vector Spaces, Second edition, Van Nostrand, Princeton, N. J., 1958.

10. - Introduction to Hilbert Space, Second edition, Van Nostrand, Princeton, N. J., 1957.

11. H. Helson, Lectures on Invariant Subspaces, Academic Press, New York, 1964.

12. E. Hille and R. S. Phillips, Functional Analysis and Semi-groups, Revised edition, Amer. Math. Soc. Colloq. Publ., Vol. 31, Amer. Math. Soc., Providence, R. I., 1957.

13. L. Hormander, An Introduction to Complex Analysis in Several Variables, Van Nostrand, Princeton, N. J., 1967.

14. J. L. Kelley and I. Namioka, Linear Topological Spaces, Van Nostrand, Princeton, N. J., 1963.

15. M. S. Livsic, On Spectral Resolution of Linear Nonself Adjoint Operators, Mat. Sb., 34 (76), (1954), 145-199. English translation Amer. Math. Soc. Translations (2), 5 (1957), 67-114.

16. S. MacLane, Homology, Academic Press, New York, 1963.

17. M. A. Naimark, Normed Rings, P. Noordhoff N. V., Groningen, Netherlands, 1964.

18. R. R. Phelps, Lectures on Choquets Theorem, Van Nostrand Mathematical Studies 7, Van Nostrand, Princeton, N. J., 1966.

19. G. C. Rota, On models for linear operators, Comm. Pure Appl. Math., 13 (1960), 469-472.

20. V. N. Singh, Reproducing Kernels and Operators with a Cyclic Vector, Thesis, University of California, Berkeley, 1969.

Received February 27, 1973.

INDIAN STATISTICAL INSTitute 


\section{PACIFIC JOURNAL OF MATHEMATICS}

EDITORS

RICHARD ARens (Managing Editor)

University of California

Los Angeles, California 90024

\section{R. A. Beaumont}

University of Washington

Seattle. Washington 98105

\section{J. DugundjI}

Department of Mathematics University of Southern California Los Angeles, California 90007

D. Gilbarg and J. Milgram

Stanford University

Stanford. California 94305

\section{ASSOCIATE EDITORS}
E. F. BECKENBACH
B. H. NEUMANN
F. WOLF
K. Yoshida

\section{SUPPORTING INSTITUTIONS}

\author{
UNIVERSITY OF BRITISH COLUMBIA \\ CALIFORNIA INSTITUTE OF TECHNOLOGY \\ UNIVERSITY OF CALIFORNIA \\ MONTANA STATE UNIVERSITY \\ UNIVERSITY OF NEVADA \\ NEW MEXICO STATE UNIVERSITY \\ OREGON STATE UNIVERSITY \\ UNIVERSITY OF OREGON \\ OSAKA UNIVERSITY
}

\author{
UNIVERSITY OF SOUTHERN CALIFORNIA \\ STANFORD UNIVERSITY \\ UNIVERSITY OF TOKYO \\ UNIVERSITY OF UTAH \\ WASHINGTON STATE UNIVERSITY \\ UNIVERSITY OF WASHINGTON

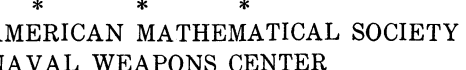

The Supporting Institutions listed above contribute to the cost of publication of this Journal, but they are not owners or publishers and have no responsibility for its content or policies.

Mathematical papers intended for publication in the Pacific Journal of Mathematics should be in typed form or offset-reproduced, (not dittoed), double spaced with large margins. Underline Greek letters in red, German in green, and script in blue. The first paragraph or two must be capable of being used separately as a synopsis of the entire paper. Items of the bibliography should not be cited there unless absolutely necessary, in which case they must be identified by author and Journal, rather than by item number. Manuscripts, in duplicate if possible, may be sent to any one of the four editors. Please classify according to the scheme of Math. Rev. Index to Vol. 39. All other communications to the editors should be addressed to the managing editor, or Elaine Barth, University of California, Los Angeles, California, 90024.

100 reprints are provided free for each article, only if page charges have been substantially paid Additional copies may be obtained at cost in multiples of 50 .

The Pacific of Journal Mathematics is issued monthly as of January 1966. Regular subscription rate: $\$ 72.00$ a year (6 Vols., 12 issues). Special rate: $\$ 36.00$ a year to individual members of supporting institutions.

Subscriptions, orders for back numbers, and changes of address should be sent to Pacific Journal of Mathematics, 103 Highland Boulevard, Berkeley, California, 94708.

\section{PUBLISHED BY PACIFIC JOURNAL OF MATHEMATICS, A NON-PROFIT CORPORATION}

Printed at Kokusai Bunken Insatsusha (International Academic Printing Co., Ltd.), 270, 3-chome Totsuka-cho. Shinjuku-ku, Tokyo 160. Japan.

Copyright (C) 1973 by Pacific Journal of Mathematics Manufactured and first issued in Japan 


\section{Pacific Journal of Mathematics}

\section{Vol. 52, No. $2 \quad$ February, 1974}

Harm Bart, Spectral properties of locally holomorphic vector-valued functions .....

J. Adrian (John) Bondy and Robert Louis Hemminger, Reconstructing infinite

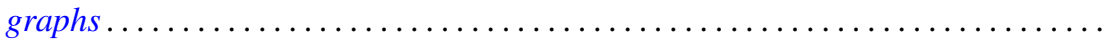

Bryan Edmund Cain and Richard J. Tondra, Biholomorphic approximation of planar

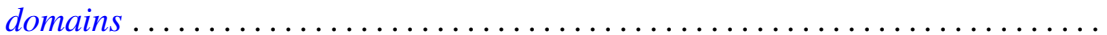

Richard Carey and Joel David Pincus, Eigenvalues of seminormal operators,

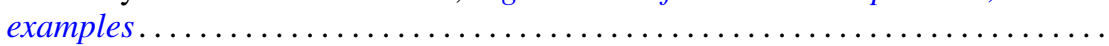

Tyrone Duncan, Absolute continuity for abstract Wiener spaces . . . . . . . . . . . . Joe Wayne Fisher and Louis Halle Rowen, An embedding of semiprime

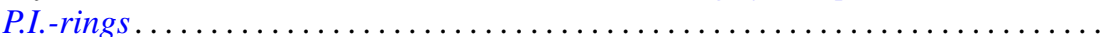

Andrew S. Geue, Precompact and collectively semi-precompact sets of semi-precompact continuous linear operators. . . . . . . . . . . . . . . .

Charles Lemuel Hagopian, Locally homeomorphic $\lambda$ connected plane continua ..... . Darald Joe Hartfiel, A study of convex sets of stochastic matrices induced by

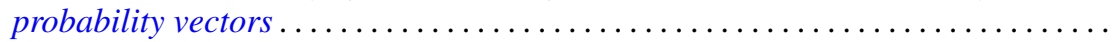

Yasunori Ishibashi, Some remarks on high order derivations $\ldots \ldots \ldots \ldots \ldots \ldots \ldots$ Donald Gordon James, Orthogonal groups of dyadic unimodular quadratic forms.

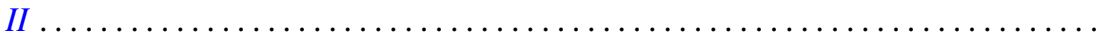

Geoffrey Thomas Jones, Projective pseudo-complemented semilattices . . . . . . . . . Darrell Conley Kent, Kelly Denis McKennon, G. Richardson and M. Schroder,

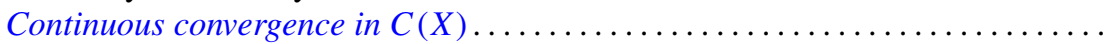

J. J. Koliha, Some convergence theorems in Banach algebras ...

Tsang Hai Kuo, Projections in the spaces of bounded linear oper

George Berry Leeman, Jr., A local estimate for typically real functions . .

475

Andrew Guy Markoe, A characterization of normal analytic spaces by the

homological codimension of the structure sheaf .........

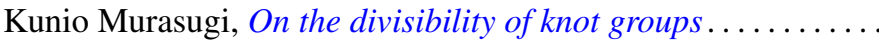

John Phillips, Perturbations of type I von Neumann algebras.

Billy E. Rhoades, Commutants of some quasi-Hausdorff matrices . .

David W. Roeder, Category theory applied to Pontryagin duality

Maxwell Alexander Rosenlicht, The nonminimality of the differential closure .

Peter Michael Rosenthal, On an inversion theorem for the general Mehler-Fock transform pair.

Alan Saleski, Stopping times for Bernoulli automorphisms

John Herman Scheuneman, Fundamental groups of compact complete locally affine complex surfaces. II. ........................

Vashishtha Narayan Singh, Reproducing kernels and operators with a cyclic vector. I. .

Peggy Strait, On the maximum and minimum of partial sums of random variables.

J. L. Brenner, Maximal ideals in the near ring of polynomials modulo 2 .

Ernst Gabor Straus, Remark on the preceding paper: "Ideals in near rings of polynomials over a field" ..........................

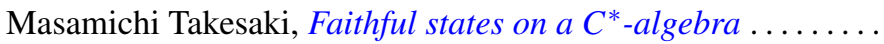

R. Michael Tanner, Some content maximizing properties of the regular simplex.

Andrew Bao-hwa Wang, An analogue of the Paley-Wiener theorem for certain

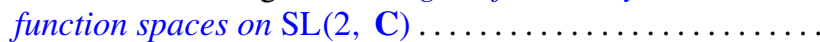

